\title{
Widely assumed phenotypic associations in Cannabis sativa lack a shared genetic basis
}

\author{
Daniela Vergara ${ }^{\text {Corresp., } 1}$, Cellene M Feathers ${ }^{1}$, Ezra L Huscher ${ }^{1}$, Ben Holmes ${ }^{2}$, Jacob A Haas ${ }^{3}$, Nolan C Kane ${ }^{1}$ \\ 1 Ebio, University of Colorado at Boulder, Boulder, Colorado, United States \\ 2 Centennial Seeds, Lafayette, Colorado, United States \\ 3 DabLogic, 5000 east 41 st ave, Denver, Colorado, USA \\ Corresponding Author: Daniela Vergara \\ Email address: daniela.vergara@colorado.edu
}

The flowering plant Cannabis sativa, cultivated for centuries for multiple purposes, displays extensive variation in phenotypic traits in addition to its wide array of secondary metabolite production. Notably, Cannabis produces two well-known secondary-metabolite cannabinoids: cannabidiolic acid (CBDA) and delta-9-tetrahydrocannabinolic acid (THCA), which are the main products sought by consumers in the medical and recreational market. Cannabis has several suggested subspecies which have been shown to differ in chemistry, branching patterns, leaf morphology and other traits. In this study we obtained measurements related to phytochemistry, reproductive traits, growth architecture, and leaf morphology from 297 hybrid individuals from a cross between two diverse lineages. We explored correlations among these characteristics to inform our understanding of which traits may be causally associated. Many of the traits widely assumed to be strongly correlated did not show any relationship in this hybrid population. The current taxonomy and legal regulation within Cannabis is based on phenotypic and chemical characteristics. However, we find these traits are not associated when lineages are inter-crossed, which is a common breeding practice and forms the basis of most modern marijuana and hemp germplasms. Our results suggest naming conventions based on leaf morphology do not correspond to the chemical properties in plants with hybrid ancestry. Therefore, a new system for identifying variation within Cannabis is warranted that will provide reliable identifiers of the properties important for recreational and, especially, medical use. 
1 Widely assumed phenotypic associations in Cannabis sativa

2 lack a shared genetic basis

3

4

5

6

7

8
Daniela Vergara ${ }^{1 *}$, Cellene M. Feathers ${ }^{1}$, Ezra L. Huscher ${ }^{1}$, Ben Holmes ${ }^{2}$, Jacob A. Haas ${ }^{1,3}$, and Nolan C. Kane ${ }^{1 *}$

1. University of Colorado, Boulder, Department of Ecology and Evolutionary Biology

2. Centennial Seeds, 1216 Commerce Ct. No.7, Lafayette, CO 80026.

3. DabLogic, 5000 east 41st ave Denver C0 80216

*Correspondence: daniela.vergara@colorado.edu or nolan.kane@colorado.edu University of Colorado Boulder 1900 Pleasant Street Boulder, CO 80309

Keywords: cannabinoid, chemotype, geometric morphometrics, hemp, marijuana, shape 0

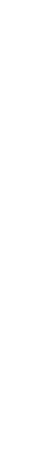


36

37

38

39

40

41

42

43

44

45

46

47

48

49

50

51

52

53

54

55

56

57

58

\section{Abstract}

The flowering plant Cannabis sativa, cultivated for centuries for multiple purposes, displays extensive variation in phenotypic traits in addition to its wide array of secondary metabolite production. Notably, Cannabis produces two well-known secondary-metabolite cannabinoids: cannabidiolic acid (CBDA) and delta-9-tetrahydrocannabinolic acid (THCA), which are the main products sought by consumers in the medical and recreational market. Cannabis has several suggested subspecies which have been shown to differ in chemistry, branching patterns, leaf morphology and other traits. In this study we obtained measurements related to phytochemistry, reproductive traits, growth architecture, and leaf morphology from 297 hybrid individuals from a cross between two diverse lineages. We explored correlations among these characteristics to inform our understanding of which traits may be causally associated. Many of the traits widely assumed to be strongly correlated did not show any relationship in this hybrid population. The current taxonomy and legal regulation within Cannabis is based on phenotypic and chemical characteristics. However, we find these traits are not associated when lineages are inter-crossed, which is a common breeding practice and forms the basis of most modern marijuana and hemp germplasms. Our results suggest naming conventions based on leaf morphology do not correspond to the chemical properties in plants with hybrid ancestry. Therefore, a new system for identifying variation within Cannabis is warranted that will provide reliable identifiers of the properties important for recreational and, especially, medical use.

Words: 229

\section{Introduction}

Phenotypic variation within and between populations is an important characteristic to consider for classification purposes. In particular, if phenotypic variation exists between different lineages within a species, then characterizing trait correlations can shed light on how they are inherited, whether they are controlled by the same genes, and if they can be used for taxonomic purposes. Wild populations may carry shared ancestral traits despite being independently inherited, and therefore it may appear as if these traits were associated. Additionally, selection may favor certain trait combinations.

The angiosperm species Cannabis sativa has been cultivated for millennia for a range of purposes (Li 1973; Li 1974; Russo 2007) and is currently, by some estimates, one 
68 of the world's most valuable crops (Hutchison et al. 2019). Unfortunately, widespread legal

69 issues have hindered Cannabis research.

70 One of the most notable characteristics of the Cannabis plant is its chemistry: the

71 production of a family of molecules known as cannabinoids which are mainly produced and

72 stored in the trichomes of female flowers (Gagne et al. 2012; Sirikantaramas et al. 2005).

73 The most studied of these cannabinoids are cannabidiolic acid (CBDA), and delta-9-

74 tetrahydrocannabinolic acid (THCA), which are produced by the enzymes CBDA and THCA

75 synthases, respectively. These two synthases are found at the final stage of the biochemical

76 pathway, along with cannabichromenic acid synthase, a third less well-studied synthase,

77 that produces Cannabichomenic acid (CBCA; Page \& Stout 2017). These three synthases use

78 the same precursor molecule, cannabigerolic acid (CBGA; Laverty et al. 2019; Page \&

79 Boubakir 2014; Vergara et al. 2019). The genetic sequences for the three synthases are

80 very similar and at least the genes encoding CBDA and THCA synthase are close in

81 proximity (Weilblen et al. 2015), suggesting they may have originated from the same

82 ancestor gene (Onofri et al. 2015; Padgitt-Cobb et al. 2019). Additionally, in vitro, each of

83 these synthases can produce at least eight different compounds including THCA and CBDA

84 in different ratios (Kovalchuk et al. 2020; Zirpel et al. 2018). These enzymes may be

85 classified as "promiscuous enzymes" due to their considerable similarities, the fact they act

86 on the same precursor molecule, and they can produce each other's compounds (Auldridge

87 et al. 2006; Chakraborty et al. 2013; Franco 2011).

88 When heated, THCA and CBDA are converted into the neutral forms THC and CBD

89 (Russo 2011), which interact with the human endocannabinoid system (Pertwee 1988;

90 Pertwee 1997; Pertwee 2004). Both THC and CBD have medicinal (Russo 2011; Swift et al. 
91 2013; Volkow et al. 2014) and economic value (Evans 2013; Kirsch 2018), but THC has

92 been intensely selected by breeders and growers (Volkow et al. 2014) due to its

93 psychoactive effects (ElSohly \& Slade 2005). Studies have also found CBDA may have

94 medicinal benefits in its acidic form (Takeda et al. 2008; Takeda et al. 2012). Differences in

95 leaf size, leaf shape, plant size, and inflorescence size are used in the Cannabis industry to

96 categorize plants and these morphological differences are thought to be useful predictors

97 of cannabinoid content.

98 Currently recognized lineages within the genus Cannabis include the narrowleaf

99 drug types, C. sativa ssp. sativa, the broadleaf drug type C. sativa ssp. indica, the northern

100 Eurasian wild C. sativa ssp. ruderalis, and at least one lineage of hemp (Clarke \& Merlin 2013).

101 Among these subspecies, there is substantial phenotypic variation in the production of

102 multiple cannabinoids (McPartland \& Russo 2001; Russo et al. 2008; Russo \& McPartland

103 2003) and terpenoids (de la Fuente et al. 2020; Orser et al. 2017; Reimann-Philipp et al.

104 2019), substantial genotypic variation (Kovalchuk et al. 2020; Lynch et al. 2016; Sawler et

105 al. 2015; Vergara et al. 2016), and observed morphological variation in traits such as

106 branching, internode length, and flowering time (Clarke \& Merlin 2013).

107 The main classifications used in the modern Cannabis industry parlance are "indica",

108 "sativa", and "hybrids". Sativa plants are described as tall with narrow leaves and lighter

109 density buds, allegedly producing high levels of THCA, and therefore have uplifting and

110 stimulating psychedelic effects after consumption. Indica plants are described as short with

111 broad leaves and dense buds, and produce high levels of both THCA and CBDA believed to

112 produce a relaxing effect (Clarke \& Merlin 2013; McPartland 2017; Vergara et al. 2016).

113 Yet, the associations between these multiple traits have not previously been researched. 
114 Other popular ideas suggest the important distinctions between Cannabis lineages related

115 to the effects after consumption are due to differences in terpene profiles rather than to

116 cannabinoid profiles. It may be that terpene profiles are more relevant to differences in

117 Cannabis lineages than cannabinoids (de la Fuente et al. 2020; Orser et al. 2017; Reimann-

118 Philipp et al. 2019). Crosses between "sativa" and "indica" plants are referred to as

119 "hybrids" and these have variable phenotypes usually intermediate to the parents (Vergara

120 et al. 2016). Finally, the hemp group has been traditionally used for industrial purposes

121 such as fiber or oil production, however the legal definition of hemp includes any Cannabis

122 plant with less than $0.3 \%$ THC by weight.

123 The colloquial naming convention of "indica" and "sativa" do not correspond to the

124 scientific subspecies with similar names. Furthermore, these common distinctions do not

125 reflect evolutionary relationships (Sawler et al. 2015; Schwabe \& McGlaughlin 2019;

126 Vergara et al. 2016). This misidentification can be particularly problematic for medical

127 patients who are depending on reliable and consistent products.

128 Cannabis is dioecious (Divashuk et al. 2014; van Bakel et al. 2011), although

129 monoecious plants exist, particularly in the hemp lineage (Hillig 2005; Peil et al. 2003).

130 Dioecious varieties are common for medicinal and recreational purposes, and selection in

131 domestication has been focused on females due to the production of cannabinoids (Gagne

132 et al. 2012; Sirikantaramas et al. 2005), with strong selection against males and

133 hermaphrodites. Many commercially important traits are expressed at maturity, and if

134 breeders could predict their late-stage expression through correlations among these traits

135 earlier during development, selection could be made sooner, accelerating breeding cycles.

136 Also, if traits early in the development allowed for distinguishing between sexes, males 
137 could be culled before pollen production and potential female pollination. This is important

138 because females would undesirably divert energy to seeds instead of cannabinoids after

139 being pollinated (Clarke \& Merlin 2013).

140 In this study, we quantified 18 phenotypic traits of 297 individuals from a first-

141 generation backcross (BC1) between a female 'Carmagnola' hemp and a male marijuana-

142 type plant 'Afghan Kush'. Many of the morphological traits we measured are considered

143 important by the Cannabis industry to characterize different plants. We predicted these

144 traits would vary in the $\mathrm{BC} 1$, and therefore allow us to investigate the pattern of possible

145 genetic correlations. Furthermore, we determined whether the association between

146 morphological traits and cannabinoid chemistry could be used to characterize Cannabis

147 lineages. Given that the current nomenclature is not supported scientific research (Lynch et

148 al. 2016; Sawler et al. 2015; Vergara et al. 2016), it is possible that erroneous associations

149 between morphological traits has contributed to the misnaming issues in the Cannabis

150 industry.

\section{Methods}

153 BC cross and measurements

154 A cross was performed between a female, narrowleaf 'Carmagnola' hemp plant and

155 a male broadleaf 'Afghan Kush' plant at Centennial Seeds in Lafayette Colorado (Figure 1A).

156 One of the female first filial (F1) offspring of this cross was backcrossed to a male sibling of

157 the parental male (Figure 1A). Two hundred ninety-seven individuals from this backcross

158 population (BC1; Figure 1A) were started indoors on April 23, 2015 and on June 6, 2015, 
159 when the plants were six weeks old, they were transplanted outdoors in a field in Boulder 160 County, Colorado.

161 Morphological measurements including height, stalk diameter, inner-node length,

162 petiole length, leaf length and width, among other measurements, were obtained at two

163 different time points during the growing cycle (Table S1). We chose these two time points,

164 one at the beginning and one at the end of the growing season, to provide information on

165 possible trait associations during the plant's development. The initial timepoint (IT) was

166 taken at six weeks old (June 6, 2015), and the final timepoint (FT) at 19 weeks old

167 (September 2, 2015) which corresponds to the beginning of the fall season. Additional

168 traits were measured at the FT including bud count, size of biggest bud, length of longest

169 branch, and number of buds on the longest branch (Table S1). At the FT, we also scanned a

170 representative leaf from each plant. Therefore, we had fully extended longest leaf (FELL)

171 measurements from both the IT and FT. At the FT, we determined the sex of all 297

172 individuals, and also measured the concentrations of three cannabinoids -THC, CBD, and

173 CBG- from 100 plants. Here we removed plants identified as male to avoid pollination, after

174 the representative leaf was scanned. Therefore, some of the measurements were taken on

175 fewer individuals than at the IT.

176

177 Phenotypic trait statistical analyses

178 To understand the change in individual phenotypes through the growing season, we

179 calculated the difference between the initial measurements and the final measurements

180 (delta $\Delta$ ) for some traits. Specifically, we calculated $\Delta$ for the four traits that were measured

181 at both timepoints (Table S1). We then use these data to estimate the within-time point 
182 correlations for both the IT and the FT, and between-time correlations. All correlations

183 were corrected with Bonferroni for multiple comparisons (Weisstein 2004). Finally, we

184 established whether any of the measured traits differed between males and females using

185 t-tests with sex as the explanatory variable.

186

187 Leaf shape analysis

188 We carried out a geometric morphometric analysis to develop a quantifiable

189 measure of leaf shape. Specifically, we placed landmark coordinates on each leaf picture

190 with the program TPS Dig2 (Rohlf 2006). We used ten landmarks from the first, central,

191 and last leaflet (Figure 1B) which covers the whole leaf structure. Additionally, we

192 measured the length and width of each of the leaves, counted the serration number in the

193 center leaflet, and counted the number of leaflets.

194 We used the R package Geomorph (Adams \& Otárola-Castillo 2013) for all geometric

195 morphometric analysis, following the methodology of Vergara et al. (2017b). A Procrustes

196 analyses was used to remove variability caused by position, orientation, and size and to

197 quantify shape variation by superimposing the objects in a joint coordinate system. Then, a

198 Principal Component Analysis (PCA) was used to identify the orthogonal structure in the

199 data and to visually explore morphological variation among individuals.

200 We performed multiple statistical tests to understand whether leaf shape was

201 related to any of the other measured traits at both timepoints and between timepoints.

202 First, we implemented several multivariate analyses of variance (MANOVAs) with shape as

203 the response variable for each of the measured traits in both timepoints and $\Delta$. We then

204 performed MANOVA models within each timepoint and $\Delta$ to understand whether the main 
205 effects of each trait affected leaf shape. We corroborated the results using multivariate 206 multiple regressions.

Cannabinoid concentration measurements

The concentrations for the three cannabinoids -- CBG, THC, and CBD -- were measured using gas chromatography on an SRI 86106 equipment with an MXT-35 column 211 using 197-209 mg of dried flower as described in (Brenneisen \& ElSohly 1988). When 212 heated, the acidic compounds CBGA, THCA, and CBDA are turned into the neutral forms

$213 \mathrm{CBG}$, THC, and CBD, which is the reason why gas chromatography only quantifies the 214 neutral forms of the compounds.

215 Given that the production of these three cannabinoids may be correlated because

216 they are part of the same biochemical pathway (Page \& Boubakir 2014; Page \& Stout 2017;

217 Vergara et al. 2019) and both CBDA and THCA synthases compete for the same precursor

218 molecule -CBGA-, we analyzed them using a Principal Component Analysis (PCA) to account

219 for multicollinearity and to avoid redundancies. We used a K-means cluster analysis on PC1 vs PC2 to visualize the different cannabinoid groups. We also added the total cannabinoid concentration and measured the ratio of each cannabinoid over this total concentration 222 (Table S1).

Statistical analyses

We examined the associations between the production of each cannabinoid and each of the measured traits at both timepoints and the $\Delta$. We also used cannabinoids as the explanatory variables for several MANOVA models to determine whether cannabinoid 
228 production explained differences among the measured traits. We also corroborated the

229 MANOVA results with multivariate multiple regressions, and correlated leaf shape to

230 cannabinoid content to understand whether any association exists between those traits.

231 Finally, we generated a variance-covariance matrix to establish the association within and

232 between all phenotypic traits.

233 These data were added to the dryad repository

234 (https://doi.org/10.5061/dryad.6t1g1jwxh). Statistical analyses, including leaf geometric 235 morphometrics, were done using R (Team 2013) and the associated code is available on

236 github (https://bit.ly/38DpE8D). All figures were generated in the R Studio platform

237 version 1.1.383 (Team 2013) and enhanced with Adobe Illustrator 2019 (v23.0.6).

239

240

241

242

243

244

245

246

247

248

249

250

251

\section{Results}

Phenotypic trait statistical analyses (including males and females)

Our results show that some phenotypic traits from the IT (Table S2) are correlated with each other after correcting with Bonferroni for multiple comparisons. For example, height is significantly correlated to the number of branches and the number of nodes even though these two traits are not significantly correlated to each other (Table S2). The positive correlation between traits related to height such as number of nodes and number of branches is expected. In other words, it is expected that tall plants will have multiple branches and nodes. It is also expected that traits that are not related to height, such as leaf-related characteristics, lack a significant correlation.

Similarly, the FT also shows that some traits are correlated at this stage (Table S3). Some of the height-related traits show a significant correlation. For example, tall plants 
252 have long side branches as well as thicker stalks. However, as expected, some traits lack 253 association, such as stalk diameter and inflorescence number or size.

254 However, many of the significant associations within either the IT or FT are lost

255 when both timepoints are correlated between them (Table S4). These various phenotypic

256 traits are not predictive between time periods (Table S4); whether a young plant is tall or

257 short is not indicative of the adult plant's height, and thus plants that are tall at the IT are

258 not always the same ones that are tall at the FT. Therefore, we cannot establish whether,

259 for example, tall plants also have thick stalks and numerous nodes since traits which are

260 correlated while young, are not significantly correlated while adults. In other words, the

261 plants exhibited different patterns of growth, irrespective of their initial size at the beginning of

262 the growing season. The lack of correlations between the timepoints suggests that some of

263 the statistically significant correlations may not be due to true biological variation but

264 instead due to chance and to the multiple comparisons, despite correcting with Bonferroni.

265 The lack of significance between the $\Delta$ correlations when compared to either the IT

266 or FT (Tables S2 and S3) suggest that some of these correlations may be spurious. The non-

267 significant correlations between the traits and their $\Delta$ indicates that the measured

268 characteristics do not follow a trend as they change over time. Therefore, the changes

269 during the plant's lifetime impede future phenotypic predictions and the initial plant

270 measurements cannot be used as an indication of future success as an adult, or how the

271 phenotype will change during the plant's lifetime.

272 Similarly, these phenotypic traits are not different between males and females

273 (Table S5). In other words, males cannot be distinguished from females with any of the

274 physical characteristics that we measured in this study. However, some trait correlations 
275 do differ between the sexes (Table S6), but again are not consistent between the

276 timepoints. The only significantly different trait between both groups is the number of

277 buds in the main branch, which was taken at the FT, where males have a larger average

278 number of buds (35.75) compared to females (24.80). However, this comparison is

279 between only four males and 19 females, as most most males were removed from the field

280 before these measurements were taken, and therefore this result may again not hold any

281 true biological meaning.

282

283 Leaf shape analysis

284 Our geometric morphometric analysis on leaf shape revealed that $82.3 \%$ of all

285 variation in leaf shape is explained by the two first principal components (Figure 2). The

286 deformation grids in the top left and bottom right corners show the extreme trends in leaf

287 morphologies. Even though these morphologies are not seen in any individual plant, these

288 are the tendencies of the leaves in these furthest points of the morphospace. The

289 individuals on the top-left side of the morphospace tend to have shorter and broader

290 leaves, and as seen in the deformation grid, the first and last leaflets are pointing outwards.

291 On the other hand, individuals in the bottom right side of the morphospace tend to have a

292 longer middle leaflet, and the first and last leaflets are clumped together pointing

293 downward. Our morphometric analysis shows no significant relationship between leaf

294 shape and the plant's sex. Therefore, both male and female plants can have similar leaf 295 shapes.

296 Our results suggest there are some trait correlations that describe leaf shape, but

297 these are not correlated to growth rates, plant size, branching architecture, 
298 phytochemistry, or plant sex (Figure S1). It appears that there could be a within-leaf effect

299 because the FELL measurements correlate within them in the IT, and serration, leaf length,

300 and number of leaflets correlate with leaf shape in the FT. However, the leaf measurements

301 show no association between timepoints (Table S7).

302 The overall trend shows leaf shape is not explained by any of the plant traits

303 measured on either timepoint (Table S7). The lack of association between a particular leaf

304 shape and plant height, or any of the other plant traits, suggests that a tall plant can have

305 broad or narrow leaves or high or low cannabinoid levels.

306 Furthermore, the MANOVA models with leaf shape as the response variable (PC1

307 and PC2, Table S7) show that the only traits that may explain leaf shape correspond to

308 other leaf measurements which are leaf length, serration, and the number of leaflets from

309 that same leaf at the FT (Figure S1). These MANOVA results confirm the within-leaf effect

310 where these measured leaf traits are associated within the same leaf. The additional

311 models that include the main effects of multiple traits support the individual MANOVA

312 results given that no significant trend in any other phenotype explains leaf shape in the IT.

313 The FT results of the within-leaf effect are confirmed with a further model including all of

314 the leaf traits (leaf length: Wilk's $\lambda=0.975, \mathrm{~F}=3.560, \mathrm{P}=0.029740$; serration: Wilk's $\lambda=$

315 0.967, F= 4.625, $\mathrm{P}<0.012$; no. leaflets Wilk's $\lambda=0.8105, \mathrm{~F}=32.483, \mathrm{P}=2.1 \mathrm{e}-13$ ). However,

316 the only significant interaction effect was between leaf length and leaf width (Wilk's $\lambda=$

$3170.967, \mathrm{~F}=4.739, \mathrm{P}<0.00095)$. The multivariate multiple regressions confirmed the

318 MANOVA results.

319

320 Cannabinoid concentration measurements 
321 Two clear chemotype clusters were identified in the PCA of cannabinoid chemistry

322 determined by the antagonistic relationships between THC versus CBD and CBG (Figure 3).

323 PC1 and PC2 explain $94.2 \%$ of the variation and the two distinct groups identified in figure

3243 (represented in triangles and diamonds) covary in a pronounced way, which show

325 covariation of the original variables with two clear chemotype clusters (Figure S2).

326 The positive loadings of both CBD and CBG on PC1 (0.71 and 0.70, respectively)

327 compared to the negative loading of THC $(-0.02)$ indicates that when CBD and CBG tend to

328 increase, THC decreases and covaries in a different direction. Therefore, the value of PC1

329 increases when CBG or CBD increase. However, on PC2, both CBG and THC have a negative

330 loading ( -0.31 and -0.91 respectively), indicating a high association, while CBD has a

331 positive loading (0.28). PC2 is primarily determined by THC given its high loading value. In

332 both PC1 and PC2, CBD and THC go in different directions.

333 Cannabinoid content showed no correlation with any of the other measured

334 phenotypic traits at either time point (IT or FT) nor with the $\Delta \mathrm{s}$ (Table S8). These results

335 were confirmed with the MANOVAs and multivariate multiple regressions.

336

337 Leaf shape vs cannabinoid content

338 We found no relationship between leaf shape and cannabinoid content using PC1 for

339 leaf shape and PC1 for cannabinoid variation (Figure 4). Therefore, leaf shape is not

340 predictive of cannabinoid content, and individuals that are high in a particular cannabinoid

341 can have elongated or short leaves. 


\section{Discussion}

343

344

345

346

347

348

349

350

351

352

353

354

355

356

357

In this study we examined correlations among various phenotypic traits from a morphologically diverse first-generation backcross (BC1) population to understand whether these multiple traits covaried with each other. We interpret these patterns of correlations as being genetically-based due to our development of a diverse array of progeny of known parentage all grown in a common environment. Our results suggest these traits are not constrained by strong genetic correlations and the initial associations between the various morphological traits in the parent generation can be broken by recombination. The lack of apparent genetic correlation between these traits suggest they can be selected for independently. Therefore, these traits are inherited independently, and would evolve separately unless selection acts to increase or maintain correlations among them. The lack of genetic correlations between the morphological traits, was also reflected in a variable pattern of growth across the growing season. The dramatic trait changes and the distribution variation over the course of plant growth and development (Coleman et al. 1994) may explain the lack of correlations between the two timepoints. This lack of correlation could also signify phenotypic plasticity which is common in plants and may be a form of adaptation (Schlichting 1986; Sultan 1995). Finally, in other species such as sugarcane, yield has not been associated to characteristics in the stalk (ie. length, weight, diameter, number) nor to other traits such as plant height (Aitken et al. 2008; Kang et al. 1983), so these patterns are likely not unique to Cannabis.

The lack of sexual dimorphism in the measured traits for this study may be specific to this population, and particularly the measurements in the FT may be problematic due to the lack of males. Theoretical models suggest differences between males and females 
365 particularly in wind-pollinated plants (Friedman \& Barrett 2009). Additionally, sex

366 differences in Cannabis have been found in traits not measured here, such as

367 photosynthetic rates and senescence (Dzhaparidze 1969; Geber et al. 2012). Future studies

368 may include these traits to examine differences between the sexes.

369 The PCA analysis facilitates the examination of shape variation for each structure

370 independently (Adams et al. 2004), allowing us to distinguish differences in leaf shape

371 (Figures 1B and 2). As size is removed during the Procrustes superimposition, it does not

372 determine the variation of the first principal component (PC1) as it does in traditional

373 morphometrics, assuring that the main source of variation explored is shape. With this

374 geometric morphometric analysis, we found that leaf shape is not related to sex (Figure 2),

375 cannabinoid production (Figure 4), or to multiple other phenotypic traits (Table S8),

376 suggesting all of these traits segregate independently. However, we did find within-leaf

377 associations between shape, leaf length, serration, and number of leaflets (Figure S1)

378 suggesting that within a single leaf some characteristics may be related to each other.

379 Because THC and CBD have attracted the most research and popular attention of all

380 the cannabinoids and their synthases both compete for the same precursor CBGA, the

381 relationship between these compounds revealed significant patterns. PC1 and PC2 for

382 cannabinoid variation (Figure 3) explain $94.2 \%$ of the variation due to the fact that there

383 are only three variables that compose the original matrix. We used a principle component

384 analysis because of the high association between these enzymes which compete for the

385 same precursor molecule (Page \& Boubakir 2014; Page \& Stout 2017), have similar

386 chemical structures (Brenneisen 2007; Flores-Sanchez \& Verpoorte 2008) and genetic

387 sequences (Onofri et al. 2015; Vergara et al. 2019), and may exemplify "sloppy enzymes" 
388 (Auldridge et al. 2006; Chakraborty et al. 2013; Franco 2011). Our results show that

389 despite the evident competition for the same precursor, as seen with the negative

390 correlation between THC and CBD (Figure S2C), all of these compounds can be present

391 together. Additionally, CBG is always seen in lower levels when compared to THC and CBD

392 (Figure S2), implying that THCA and CBDA synthases are efficiently converting CBGA into

393 THCA and CBDA respectively in this population.

394 Studies suggest that THC has been selected for by breeders and growers and that

395 varieties have been bread for higher THC potency (ElSohly et al. 2016; Volkow et al. 2014).

396 Our results confirm these studies given that THC is always produced in higher quantities

397 than CBD (Figure S2), implying that THCA synthase may be a better competitor than CBDA

398 synthase in this population.

Variation in THC production is probably a result of gene sequence variation (Onofri et al. 2015), expression levels, and gene copy number variation (Vergara et al. 2019), and

401 there are multiple genes throughout the genome associated with its production (Grassa et

al. 2018; Laverty et al. 2019). However, expression of these genes could be due to

environmental effects such as cultivation conditions (Elzinga et al. 2015), which have not

yet been quantified. Even though the parent plants were grown under different conditions

than the BC1 offspring, all of the offspring were grown under the same conditions

minimizing the environmental effects on the expression of these genes.

Although some correlations among traits are significant and make biological sense,

the traits that are associated with purported groups (i.e. indica and sativa) within Cannabis

410 are due to either shared ancestry, in the case of comparisons among subspecies or other 
411 major lineages, or correlated selection, in the case of modern hybrids. In other words,

412 correlations between leaf shape and phytochemistry may not be due to causal

413 relationships, but rather because breeders have intentionally (or unintentionally) selected

414 for these trait combinations. If these traits were associated due to shared ancestry or

415 correlated selection, their association can be broken by recombination.

416 This is particularly noticeable in most of the modern cultivars which are hybrids

417 from the supposed two main groups. Therefore, our study also suggests that common

418 assumptions about associations between leaf shape and chemistry may exacerbate the

419 misnaming problems of Cannabis varieties by the industry (Sawler et al. 2015; Vergara et

420 al. 2016). Given the lack of association between cannabinoids and other morphological

421 traits, the accepted standards for categorizing Cannabis types by the industry are deeply

422 flawed because their naming convention is based on sets of traits that could be

423 disassociated to each other. Additionally, other studies have shown that name is not

424 indicative of cannabinoid potency or overall chemical composition (Elzinga et al. 2015),

425 and that varieties are grouped based on reported flavors and aromas, regardless of genetic

426 relationships misclassifying closely related individuals (de la Fuente et al. 2020). This

427 misnaming problem in the Cannabis industry for both varieties and groupings ('sativa' and

428 'indica') is greatly magnified by the fact that scientists can only study the Cannabis

429 produced by the federal government despite its inferiority in potency and diversity, and the

430 fact that it does not reflect the products distributed in consumer markets (Schwabe et al.

431 2019; Vergara et al. 2017a). It is crucial for Cannabis researchers to disseminate accurate

432 information to the public. This is not being done adequately because the scientific literature

433 is not effectively informing public policy, medical decisions, or providing correct 
434 information on harm reduction (Hutchison et al. 2019). This lack of information has major

435 ramifications for growers, breeders, regulators, and consumers, particularly for medical

436 patients who must understand what they are consuming to achieve the greatest benefit for

437 their individual needs.

438 In order to improve the quality and efficacy of the Cannabis consumed by medical

439 patients, it is important that unbiased, accurate, and precise chemotype testing should be

440 made mandatory. However, testing facilities do not have universally established standards,

441 as cannabinoid measurements vary widely across laboratories (Jikomes \& Zoorob 2018),

442 and there are no supervising institutions that oversee testing entities or their

443 methodologies, making differences in cannabinoid reporting inevitable.

445 Conclusions

The fact that most of the phenotypic traits are not genetically correlated has significant implications for both Cannabis breeders and commercial growers. If these traits are not linked, as previously thought, then it is possible to select for new combination of traits when breeding for novel varieties. This expands the possibility of generating varieties with a unique combination of traits providing unforeseen medicinal and industrial value.

451 Future breeding can be done to maximize combinations of these traits.

\section{Acknowledgments}

455 We would like to thank J. A. Fuentes for help with the morphometric analysis, figure 456 construction and for comments on the manuscript, to C. Pogoda, A. Schwabe, and R. Miller 
457 for comments on the manuscript, and to A. Holloway and two anonymous reviewers for

458 their informative comments which greatly improved this paper.

459

460 Author Contributions

461 D.V. and C.M.F. analyzed the data and wrote the first draft of the manuscript; E.L.H.

462 calculated the Gmatrix, and measured BC1 plants; B.H. donated, grew, and cared for all

463 plants-parentals, F1, and BC1; J.H. grew, cared, and measured the BC1 plants; D.V and N.C.K.

464 conceived and lead the project and measured BC1 plants. All authors contributed to

465

466

467

468

469

470

471

472

473

474

475

476

477

478

479

480

481

482

483

484

485

486

487

488

489

490

manuscript preparation.

\section{Legends Supplementary Tables and Figures}

Table S1. All data. Data used for all analyses with each plant (column 1) its sex (column 2), unique id (column 3), the IT date (column 4), measurements for the IT (columns 5-12), the FT (column 13), measurements for the FT (columns 14-21), deltas for four measurements (columns 22-25), flower weight for cannabinoid measurement (column 26), the addition of all cannabinoids (column 27), the percentage of each cannabinoid over the total (columns 28-30), the ratio of each cannabinoid to each other (columns 31-33), the PC values for the cannabinoids (columns 34-36), leaf measurements for the FT (columns 37-39), and the leaf shape PC values (columns 41-56).

Table S2 Within IT and delta $(\Delta)$ correlations. Correlations within the initial time point and $\Delta$ for the eight traits measured in time point one and the four $\Delta$ changes

Table S3 Within FT and delta $(\Delta)$ correlations. Correlations within the final time point and $\Delta$ for the eight traits measured in the final timepoint, the four $\Delta$ changes and the four leaf-morphologic traits from the leaves used for geometric morphometrics (leaflet number, serration in the middle leaflet, leaf width and leaf length).

Table S4 Between time-point correlations. Correlations between the eight traits from time-point one against the eight traits from time point two and against the four leafmorphologic traits from the leaves used for geometric morphometrics (leaflet number, serration in the middle leaflet, leaf width and leaf length). 
493

494

495

496

497

498

499

500

501

502

503

504

505

506

507

508

509

510

511

512

513

514

515

516

517

518

519

520

521

522

523

524

525

526

527

528

529

530

531

532

Table S5. t-tests between sex and phenotypic traits. t-tests between sex and the phenotypic traits measured on both timepoints including leaf shape measurements in the IT, FT, and $\Delta$.

Table S6. Difference in trait correlations between sex. Difference between the correlations of the various phenotypic traits between males and females in the IT, FT, and $\Delta$.

Table S7. MANOVA for leaf shape against all phenotypic traits. MANOVA associations between PC1 and PC2 for leaf shape against all phenotypic traits from the IT, FT, and $\Delta$.

Table S8. Correlations between three cannabinoids CBG, CBD, and THC, and all phenotypic traits. Correlations between the three cannabinoids all phenotypic traits from the IT, FT, and $\Delta$.

Table S8. GMatrix for variance and covariance between all phenotypic traits measured. Variances and covariances between all phenotypic traits measured at both timepoints.

Figure S1. PC1 for shape versus three traits. Leaf length is not correlated to PC1 (A), while serration in the middle leaflet (B) and number of leaflets (C) are marginally and significantly correlated, respectively. Each panel at the top has the deformation grids at the minimum (left) and maximum (right) values for length (A), serration in the middle leaflet (B), and number of leaflets (C). Males are shown in squares, females in open circles. The insets show the $\mathrm{p}$ and $\mathrm{r}$ values and the variance and covariance between both variables, also given in the variance-covariance matrix (Table S8).

Figure S2. Relationships between percent THC, CBD, and CBG. A and B show a positive correlation between CBG vs CBD, and THC vs CBG, respectively. $\mathbf{C}$ displays the negative linear relationship between THC and CBD. Notice the differences in the axis representing the disparity in production of the different cannabinoids. Males are shown in squares, females in open circles. The insets show the $p$ and $r$ values and the variance and covariance between both variables, also given in the variance-covariance matrix (Table S8).

\section{References}

Adams DC, and Otárola-Castillo E. 2013. Geomorph: an R package for the collection and analysis of geometric morphometric shape data. Methods in Ecology and Evolution 4:393-399.

Adams DC, Rohlf FJ, and Slice DE. 2004. Geometric morphometrics: ten years of progress following the 'revolution'. Italian Journal of Zoology 71:5-16.

Aitken K, Hermann S, Karno K, Bonnett G, Mclntyre L, and Jackson P. 2008. Genetic control of yield related stalk traits in sugarcane. Theoretical and Applied Genetics 117:1191-1203. 
533

534

535

536

537

538

539

540

541

542

543

544

545

546

547

548

549

550

551

552

553

554

555

556

557

558

559

560

561

562

563

564

565

566

567

568

569

570

571

572

573

574

575

576

577

578

579

580

Auldridge ME, McCarty DR, and Klee HJ. 2006. Plant carotenoid cleavage oxygenases and their apocarotenoid products. Current opinion in plant biology 9:315-321.

Brenneisen R. 2007. Chemistry and analysis of phytocannabinoids and other Cannabis constituents. Marijuana and the Cannabinoids: Springer, 17-49.

Brenneisen R, and ElSohly MA. 1988. Chromatographic and spectroscopic profiles of Cannabis of different origins: Part I. Journal of Forensic Science 33:1385-1404.

Chakraborty S, Minda R, Salaye L, Dandekar AM, Bhattacharjee SK, and Rao BJ. 2013. Promiscuity-based enzyme selection for rational directed evolution experiments. Enzyme Engineering: Methods and Protocols:205-216.

Clarke R, and Merlin M. 2013. Cannabis: evolution and ethnobotany: Univ of California Press.

Coleman JS, McConnaughay KD, and Ackerly DD. 1994. Interpreting phenotypic variation in plants. Trends in Ecology \& Evolution 9:187-191.

de la Fuente A, Zamberlan F, Ferrán AS, Carrillo F, Tagliazucchi E, and Pallavicini C. 2020. Relationship among subjective responses, flavor, and chemical composition across more than 800 commercial cannabis varieties. Journal of Cannabis Research 2:1-18.

Divashuk MG, Alexandrov OS, Razumova OV, Kirov IV, and Karlov GI. 2014. Molecular cytogenetic characterization of the dioecious Cannabis sativa with an XY chromosome sex determination system. Plos One 9:e85118.

Dzhaparidze L. 1969. Sex in plants, part II. Biochemical and physiological sex differences in dioecious plants: problem of influencing sex formation. Jerusalem: IPST Press.

EISohly MA, Mehmedic Z, Foster S, Gon C, Chandra S, and Church JC. 2016. Changes in cannabis potency over the last 2 decades (1995-2014): analysis of current data in the United States. Biological psychiatry 79:613-619.

EISohly MA, and Slade D. 2005. Chemical constituents of marijuana: the complex mixture of natural cannabinoids. Life sciences 78:539-548.

Elzinga S, Fischedick J, Podkolinski R, and Raber J. 2015. Cannabinoids and terpenes as chemotaxonomic markers in cannabis. Nat Prod Chem Res 3:2.

Evans D. 2013. The economic impacts of marijuana legalization.

Flores-Sanchez IJ, and Verpoorte R. 2008. Secondary metabolism in cannabis. Phytochemistry reviews 7:615-639.

Franco OL. 2011. Peptide promiscuity: an evolutionary concept for plant defense. FEBS letters 585:9951000.

Friedman J, and Barrett SC. 2009. Wind of change: new insights on the ecology and evolution of pollination and mating in wind-pollinated plants. Annals of Botany 103:1515-1527.

Gagne SJ, Stout JM, Liu E, Boubakir Z, Clark SM, and Page JE. 2012. Identification of olivetolic acid cyclase from Cannabis sativa reveals a unique catalytic route to plant polyketides. Proceedings of the National Academy of Sciences 109:12811-12816.

Geber MA, Dawson TE, and Delph LF. 2012. Gender and sexual dimorphism in flowering plants: Springer Science \& Business Media.

Grassa CJ, Wenger JP, Dabney C, Poplawski SG, Motley ST, Michael TP, Schwartz CJ, and Weiblen GD. 2018. A complete Cannabis chromosome assembly and adaptive admixture for elevated cannabidiol (CBD) content. bioRxiv. 10.1101/458083

Hillig KW. 2005. Genetic evidence for speciation in Cannabis (Cannabaceae). Genetic Resources and Crop Evolution 52:161-180. 10.1007/s10722-003-4452-y

Hutchison KE, Bidwell LC, Ellingson JM, and Bryan AD. 2019. Cannabis and Health Research: Rapid Progress Requires Innovative Research Designs. Value in Health.

Jikomes N, and Zoorob M. 2018. The cannabinoid content of legal cannabis in Washington state varies systematically across testing facilities and popular consumer products. Scientific Reports 8:4519.

Peer) reviewing PDF | (2020:08:51989:1:0:NEW 21 Nov 2020) 
581

582

583

584

585

586

587

588

589

590

591

592

593

594

595

596

597

598

599

600

601

602

603

604

605

606

607

608

609

610

611

612

613

614

615

616

617

618

619

620

621

622

623

624

625

626

627

Kang MS, Miller JD, and Tai P. 1983. Genetic and Phenotypic Path Analyses and Heritability in Sugarcane 1. Crop Science 23:643-647.

Kirsch J. 2018. Taxation and Welfare in the Cannabis Industry: Evidence from Colorado Edibles 20142016.

Kovalchuk I, Pellino M, Rigault P, van Velzen R, Ebersbach J, R. Ashnest J, Mau M, Schranz M, Alcorn J, and Laprairie R. 2020. The Genomics of Cannabis and Its Close Relatives. Annual review of plant biology 71.

Laverty KU, Stout JM, Sullivan MJ, Shah H, Gill N, Holbrook L, Deikus G, Sebra R, Hughes TR, and Page JE. 2019. A physical and genetic map of Cannabis sativa identifies extensive rearrangements at the THC/CBD acid synthase loci. Genome Research 29:146-156.

Li HL. 1973. An archaeological and historical account of cannabis in China. Economic Botany 28:437-448.

Li HL. 1974. Origin and use of Cannabis in Eastern Asia; Linguistic-cultural implications. Economic Botany 28:293-301. 10.1007/bf02861426

Lynch RC, Vergara D, Tittes S, White K, Schwartz CJ, Gibbs MJ, Ruthenburg TC, deCesare K, Land DP, and Kane NC. 2016. Genomic and Chemical Diversity in Cannabis. Critical Reviews in Plant Sciences 35:349-363. 10.1080/07352689.2016.1265363

McPartland JM. 2017. Cannabis sativa and Cannabis indica versus "Sativa" and "Indica". Cannabis sativa L-Botany and Biotechnology: Springer, 101-121.

McPartland JM, and Russo EB. 2001. Cannabis and cannabis extracts: greater than the sum of their parts? Journal of Cannabis Therapeutics 1:103-132.

Onofri C, de Meijer EPM, and Mandolino G. 2015. Sequence heterogeneity of cannabidiolic-and tetrahydrocannabinolic acid-synthase in Cannabis sativa L. and its relationship with chemical phenotype. Phytochemistry.

Orser C, Johnson S, Speck M, Hilyard A, and Afia I. 2017. Terpenoid Chemoprofiles Distinguish Drug-type Cannabis sativa L. Cultivars in Nevada. Natural Products Chemistry and Research 6. 10.4172/2475-7675.1000304s

Padgitt-Cobb LK, Kingan SB, Wells J, Elser J, Kronmiller B, Moore D, Concepcion G, Peluso P, Rank D, Jaiswal P, Henning J, and Hendrix DA. 2019. A phased, diploid assembly of the Cascade hop $<$ em $>$ (Humulus lupulus) </em> genome reveals patterns of selection and haplotype variation. bioRxiv:786145. 10.1101/786145

Page JE, and Boubakir Z. 2014. Aromatic prenyltransferase from Cannabis. Google Patents.

Page JE, and Stout JM. 2017. Cannabichromenic acid synthase from Cannabis sativa. Google Patents.

Peil A, Flachowsky H, Schumann E, and Weber WE. 2003. Sex-linked AFLP markers indicate a pseudoautosomal region in hemp (Cannabis sativa L.). Theoretical and Applied Genetics 107:102-109.

Pertwee RG. 1988. The central neuropharmcology of psychotropic cannabinoids. Pharmacology \& therapeutics 36:189-261.

Pertwee RG. 1997. Pharmacology of cannabinoid CB1 and CB2 receptors. Pharmacology \& therapeutics 74:129-180.

Pertwee RG. 2004. Pharmacological and therapeutic targets for $\Delta 9$ tetrahydrocannabinol and cannabidiol. Euphytica 140:73-82.

Reimann-Philipp U, Speck M, Orser C, Johnson S, Hilyard A, Turner H, Stokes AJ, and Small-Howard AL. 2019. Cannabis Chemovar Nomenclature Misrepresents Chemical and Genetic Diversity; Survey of Variations in Chemical Profiles and Genetic Markers in Nevada Medical Cannabis Samples. Cannabis and Cannabinoid Research.

Russo EB. 2007. History of cannabis and its preparations in saga, science, and sobriquet. Chemistry \& Biodiversity 4:1614-1648. 10.1002/cbdv.200790144 
628

629

630

631

632

633

634

635

636

637

638

639

640

641

642

643

644

645

646

647

648

649

650

651

652

653

654

655

656

657

658

659

660

661

662

663

664

665

666

667

668

669

670

671

672

673

674

675
Russo EB. 2011. Taming THC: potential cannabis synergy and phytocannabinoid-terpenoid entourage effects. British Journal of Pharmacology 163:1344-1364. 10.1111/j.1476-5381.2011.01238.x

Russo EB, Jiang H-E, Li X, Sutton A, Carboni A, del Bianco F, Mandolino G, Potter DJ, Zhao Y-X, Bera S, Zhang Y-B, Lue E-G, Ferguson DK, Hueber F, Zhao L-C, Liu C-J, Wang Y-F, and Li C-S. 2008. Phytochemical and genetic analyses of ancient cannabis from Central Asia. Journal of Experimental Botany 59:4171-4182. 10.1093/jxb/ern260

Russo EB, and McPartland JM. 2003. Cannabis is more than simply $\Delta 9$-tetrahydrocannabinol. Psychopharmacology 165:431-432.

Sawler J, Stout JM, Gardner KM, Hudson D, Vidmar J, Butler L, Page JE, and Myles S. 2015. The Genetic Structure of Marijuana and Hemp. Plos One 10:e0133292.

Schlichting CD. 1986. The evolution of phenotypic plasticity in plants. Annual Review of Ecology and Systematics 17:667-693.

Schwabe AL, Hansen CJ, Hyslop RM, and McGlaughlin ME. 2019. Research grade marijuana supplied by the National Institute on Drug Abuse is genetically divergent from commercially available <em>Cannabis</em>. bioRxiv:592725. 10.1101/592725

Schwabe AL, and McGlaughlin ME. 2019. Genetic tools weed out misconceptions of strain reliability in Cannabis sativa: implications for a budding industry. Journal of Cannabis Research 1:3. 10.1186/s42238-019-0001-1

Sirikantaramas S, Taura F, Tanaka Y, Ishikawa Y, Morimoto S, and Shoyama Y. 2005. Tetrahydrocannabinolic acid synthase, the enzyme controlling marijuana psychoactivity, is secreted into the storage cavity of the glandular trichomes. Plant and Cell Physiology 46:15781582.

Sultan SE. 1995. Phenotypic plasticity and plant adaptation. Acta botanica neerlandica 44:363-383.

Swift W, Wong A, Li KM, Arnold JC, and McGregor IS. 2013. Analysis of cannabis seizures in NSW, Australia: cannabis potency and cannabinoid profile. Plos One 8:e70052.

Takeda S, Misawa K, Yamamoto I, and Watanabe K. 2008. Cannabidiolic acid as a selective cyclooxygenase-2 inhibitory component in cannabis. Drug metabolism and disposition 36:19171921.

Takeda S, Okajima S, Miyoshi H, Yoshida K, Okamoto Y, Okada T, Amamoto T, Watanabe K, Omiecinski CJ, and Aramaki H. 2012. Cannabidiolic acid, a major cannabinoid in fiber-type cannabis, is an inhibitor of MDA-MB-231 breast cancer cell migration. Toxicology letters 214:314-319.

Team RC. 2013. R: A language and environment for statistical computing.

van Bakel H, Stout JM, Cote AG, Tallon CM, Sharpe AG, Hughes TR, and Page JE. 2011. The draft genome and transcriptome of Cannabis sativa. Genome Biology 12. 10.1186/gb-2011-12-10-r102

Vergara D, Baker H, Clancy K, Keepers KG, Mendieta JP, Pauli CS, Tittes SB, White KH, and Kane NC. 2016. Genetic and Genomic Tools for Cannabis sativa. Critical Reviews in Plant Sciences 35:364377. 10.1080/07352689.2016.1267496

Vergara D, Bidwell LC, Gaudino R, Torres A, Du G, Ruthenburg TC, deCesare K, Land DP, Hutchison KE, and Kane NC. 2017a. Compromised External Validity: Federally Produced Cannabis Does Not Reflect Legal Markets. Scientific Reports 7:46528.

Vergara D, Fuentes JA, Stoy KS, and Lively CM. 2017b. Evaluating shell variation across different populations of a freshwater snail. Molluscan Research 37:120-132.

Vergara D, Huscher EL, Keepers KG, Givens RM, Cizek CG, Torres A, Gaudino R, and Kane NC. 2019. Gene copy number is associated with phytochemistry in <em>Cannabis sativa</em>. bioRxiv:736181. 10.1101/736181

Volkow ND, Baler RD, Compton WM, and Weiss SRB. 2014. Adverse Health Effects of Marijuana Use. New England Journal of Medicine 370:2219-2227. 10.1056/NEJMra1402309

Weisstein EW. 2004. Bonferroni correction. https://mathworld wolfram com/. 
676

677

678

679

680

681

682

683

684
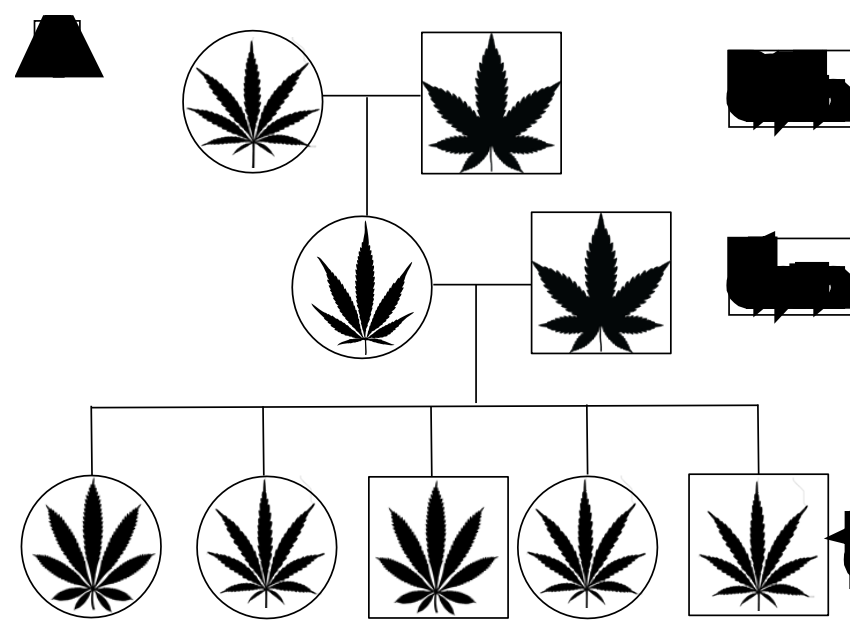

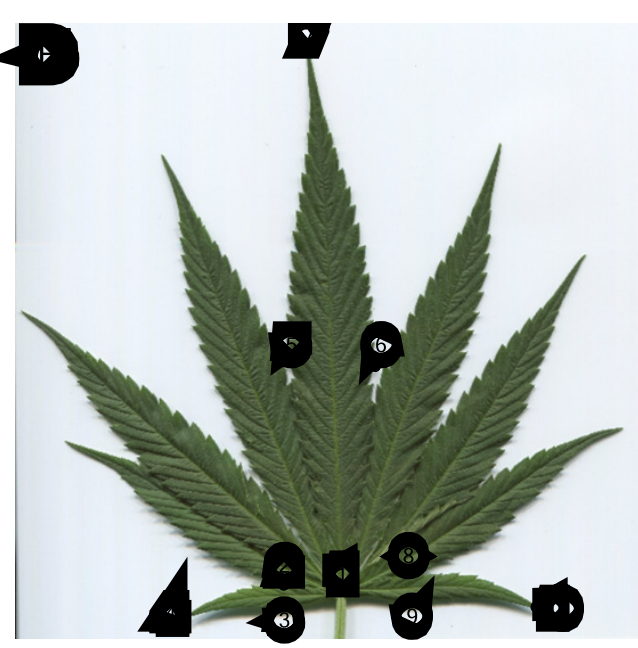

685

686

687

688

689

690
Figure 1. Pedigree and landmarks. Panel A: Pedigree of a first-generation backcross (BC1) between a male marijuana-type Afghan Kush and a female Carmagnola hemp. The F1 generation was backcrossed with a brother from the original male Afghan Kush to produce the BC1 Generation. Panel B: Exemplar leaf depicting the ten points used for leaf shape analysis. The ten points measured the first, central, and last leaflets. 


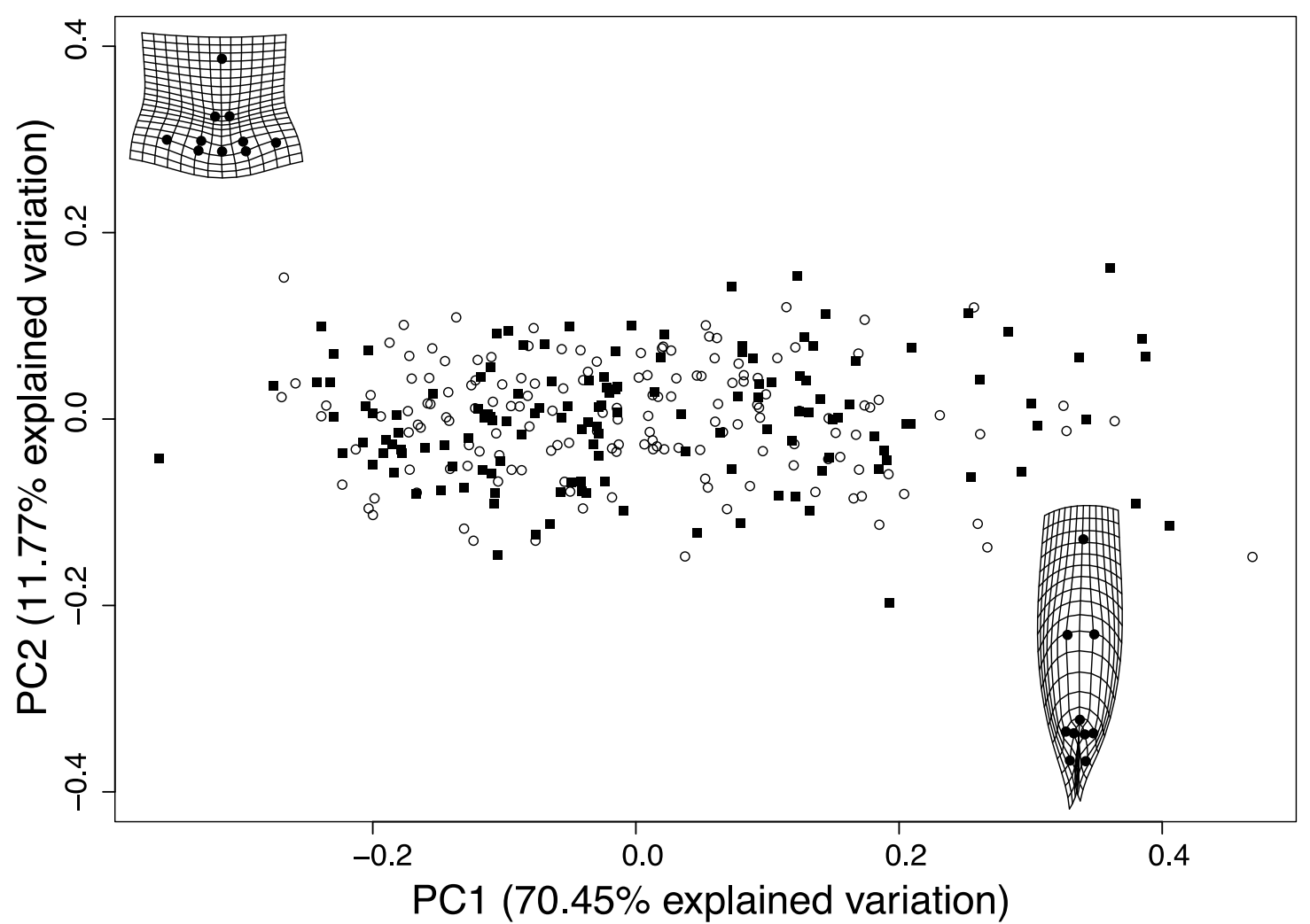

691

692

693

694

695

696

697

698

699

700
Figure 2: Geometric morphometric analysis of leaf shape. The two first PC explain $82.3 \%$ of the leaf shape variation, which is not related to sex (males are squares, females are open circles). The deformation grids mostly show the leaf deformations on PC1 which contains most of the variation. The grids show that individuals in the top left have squatter, broader leaves with the first and last leaflets pointing outwards, while those on the bottom right side of the morphospace have thinner leaves with a long mid-leaflet and the two outer leaflets lumped together pointing downwards. 


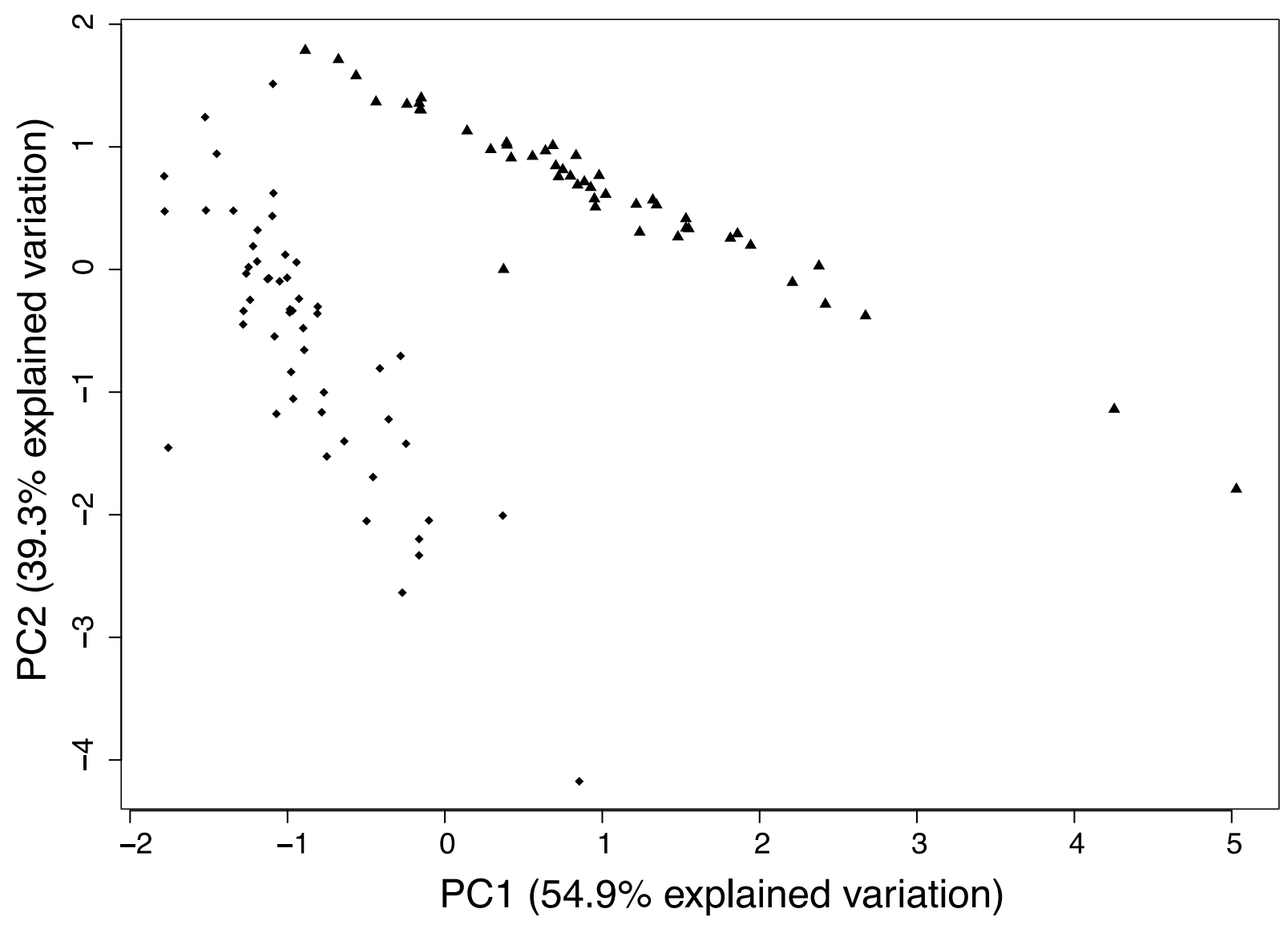

Figure 3. PC1 and PC2 for cannabinoid variation. PC1 and PC2 explain $94.2 \%$ of the overall cannabinoid variation for the three cannabinoids measured. There are two clear groups in the graph, squares and triangles, which correspond to the two clear cannabinoid clusters. The overall trend shows that the squares 705 have low CBG and CBD and high THC. The triangles show high CBD and low THC.

707 


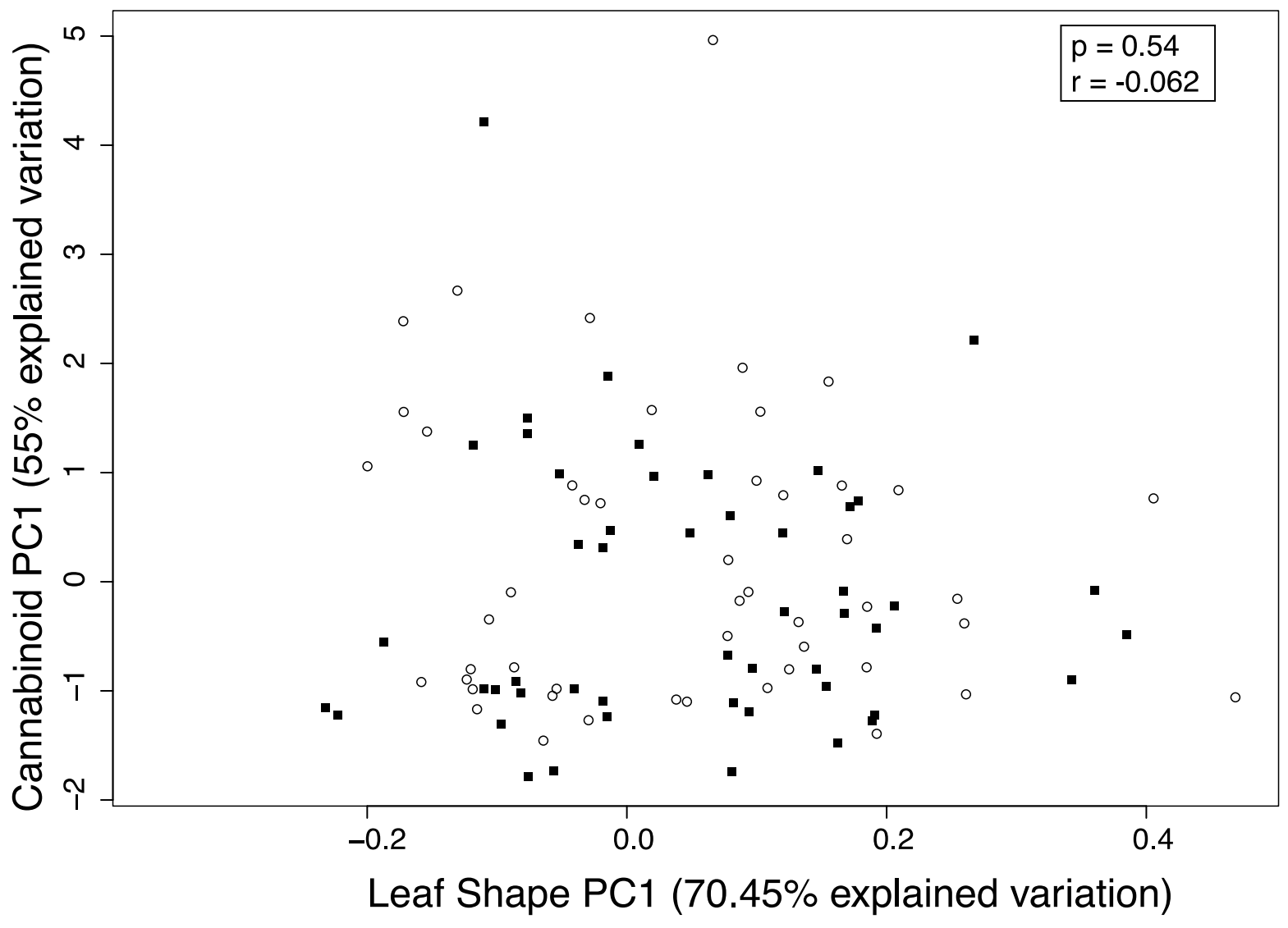

709 Figure 4: Correlation between leaf shape (PC1) and cannabinoid variation (PC1). Leaf shape is not

710 significantly to cannabinoid variation even at the most extreme points in the morphospace. Males are shown 711 in squares and females in open circles.

712 
Figure 1

Figure 1. Pedigree and landmarks.

Panel A: Pedigree of a first-generation backcross $(B C 1)$ between a male marijuana-type Afghan Kush and a female Carmagnola hemp. The F1 generation was backcrossed with a brother from the original male Afghan Kush to produce the BC1 Generation. Panel B:

Exemplar leaf depicting the ten points used for leaf shape analysis. The ten points measured the first, central, and last leaflets.
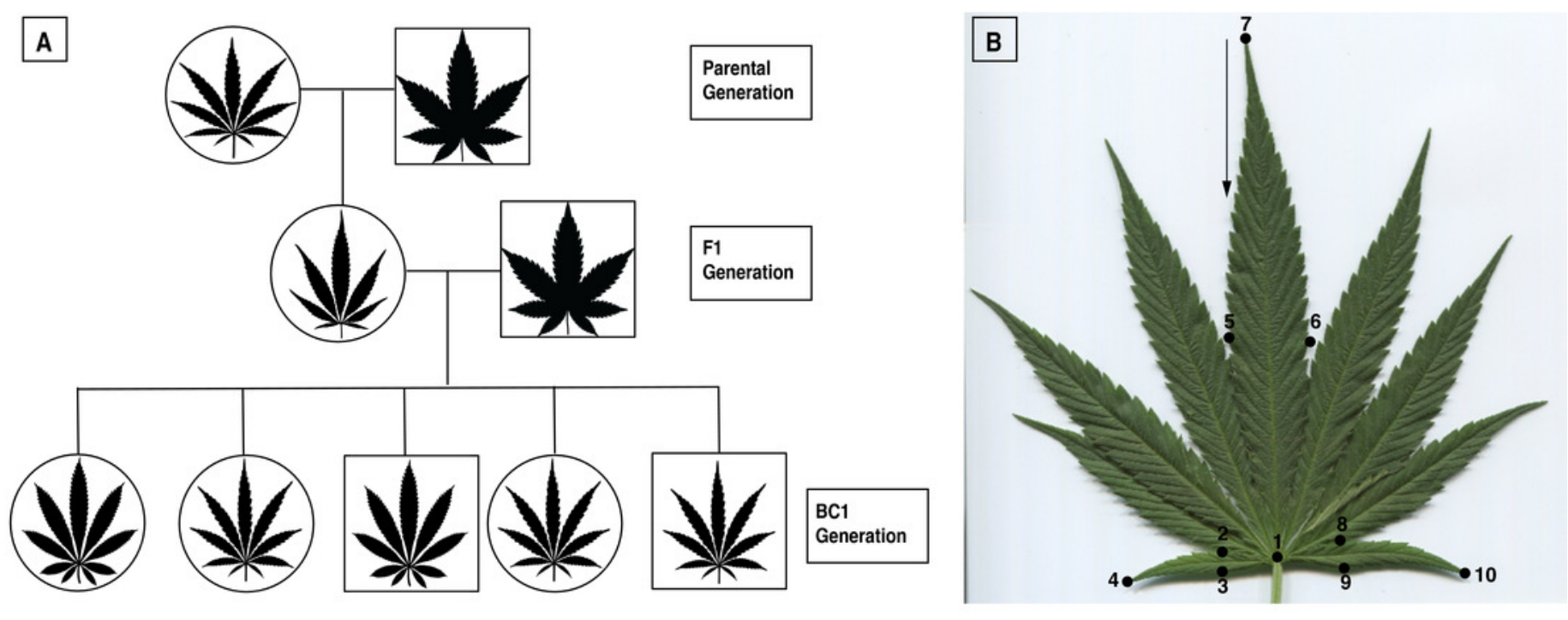
Figure 2

Figure 2: Geometric morphometric analysis of leaf shape.

The two first PC explain $82.3 \%$ of the leaf shape variation, which is not related to sex (males are squares, females are open circles). The deformation grids mostly show the leaf deformations on PC1 which contains most of the variation. The grids show that individuals in the top left have squatter, broader leaves with the first and last leaflets pointing outwards, while those on the bottom right side of the morphospace have thinner leaves with a long mid-leaflet and the two outer leaflets lumped together pointing downwards.

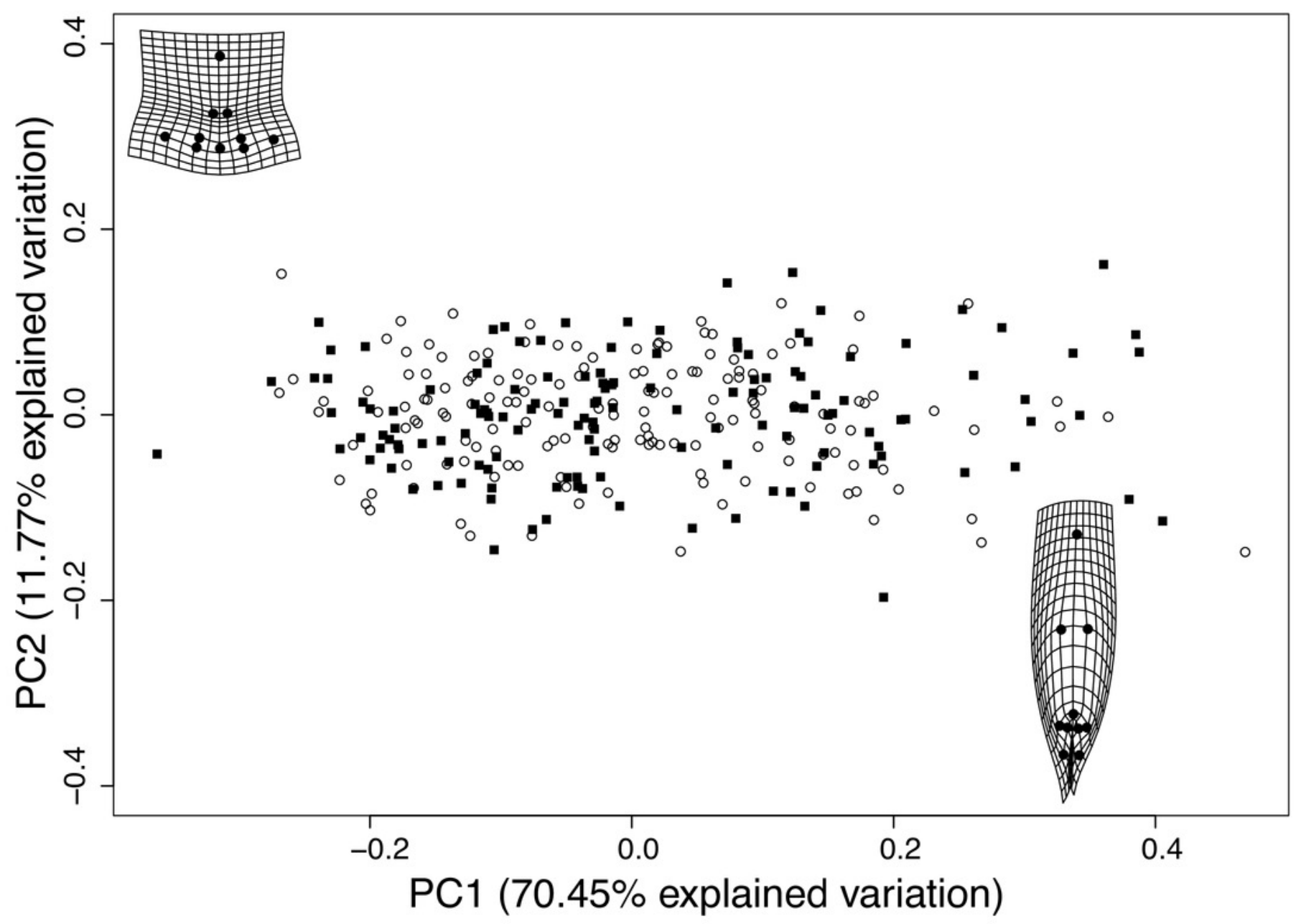


Figure 3

Figure 3. $\mathrm{PC} 1$ and $\mathrm{PC2}$ for cannabinoid variation.

PC1 and PC2 explain $94.2 \%$ of the overall cannabinoid variation for the three cannabinoids measured. There are two clear groups in the graph, squares and triangles, which correspond to the two clear cannabinoid clusters. The overall trend shows that the squares have low CBG and CBD and high THC. The triangles show high CBD and low THC.

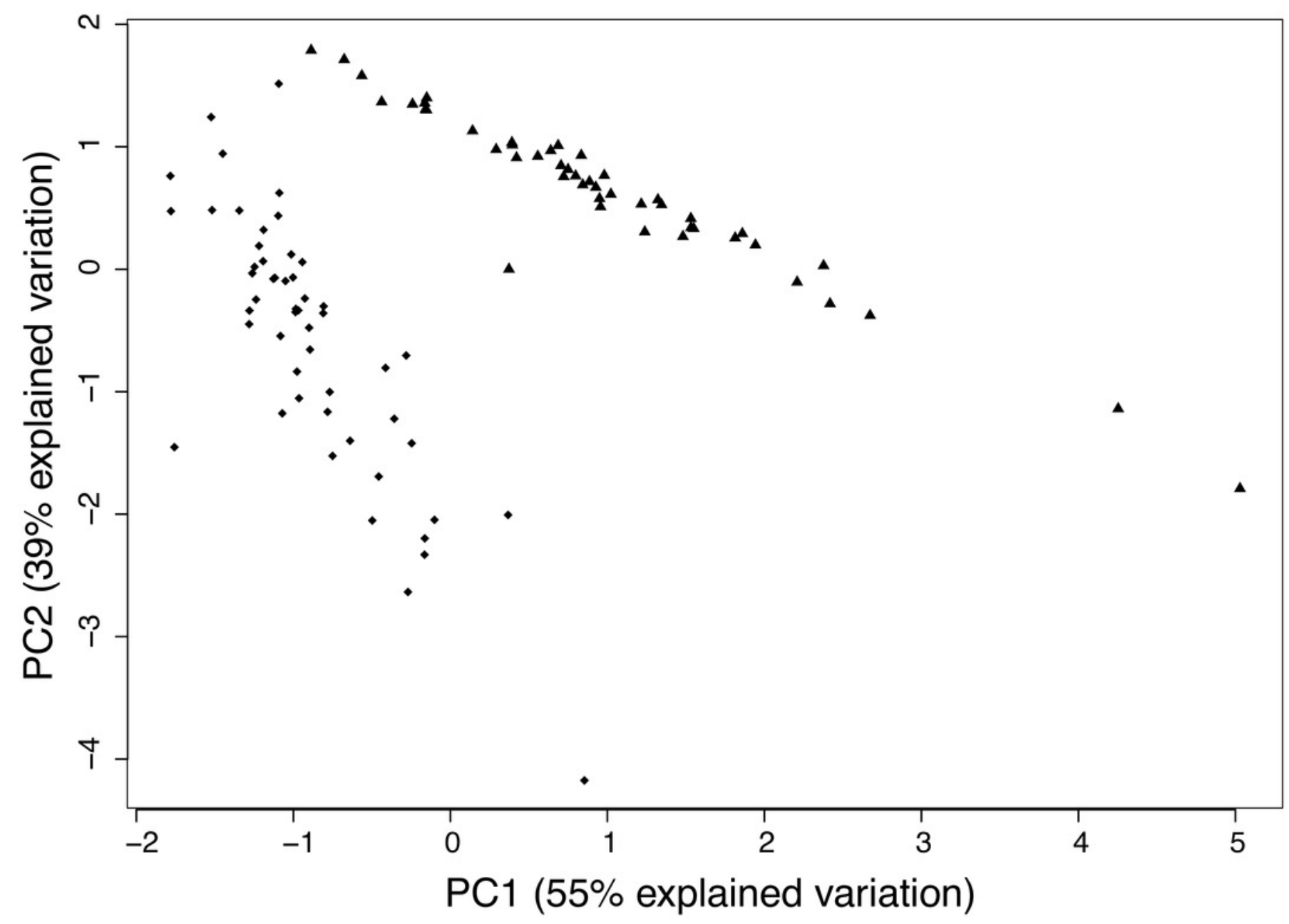


Figure 4

Figure 4: Correlation between leaf shape (PC1) and cannabinoid variation (PC1).

Leaf shape is not significantly to cannabinoid variation even at the most extreme points in the morphospace. Males are shown in squares and females in open circles.

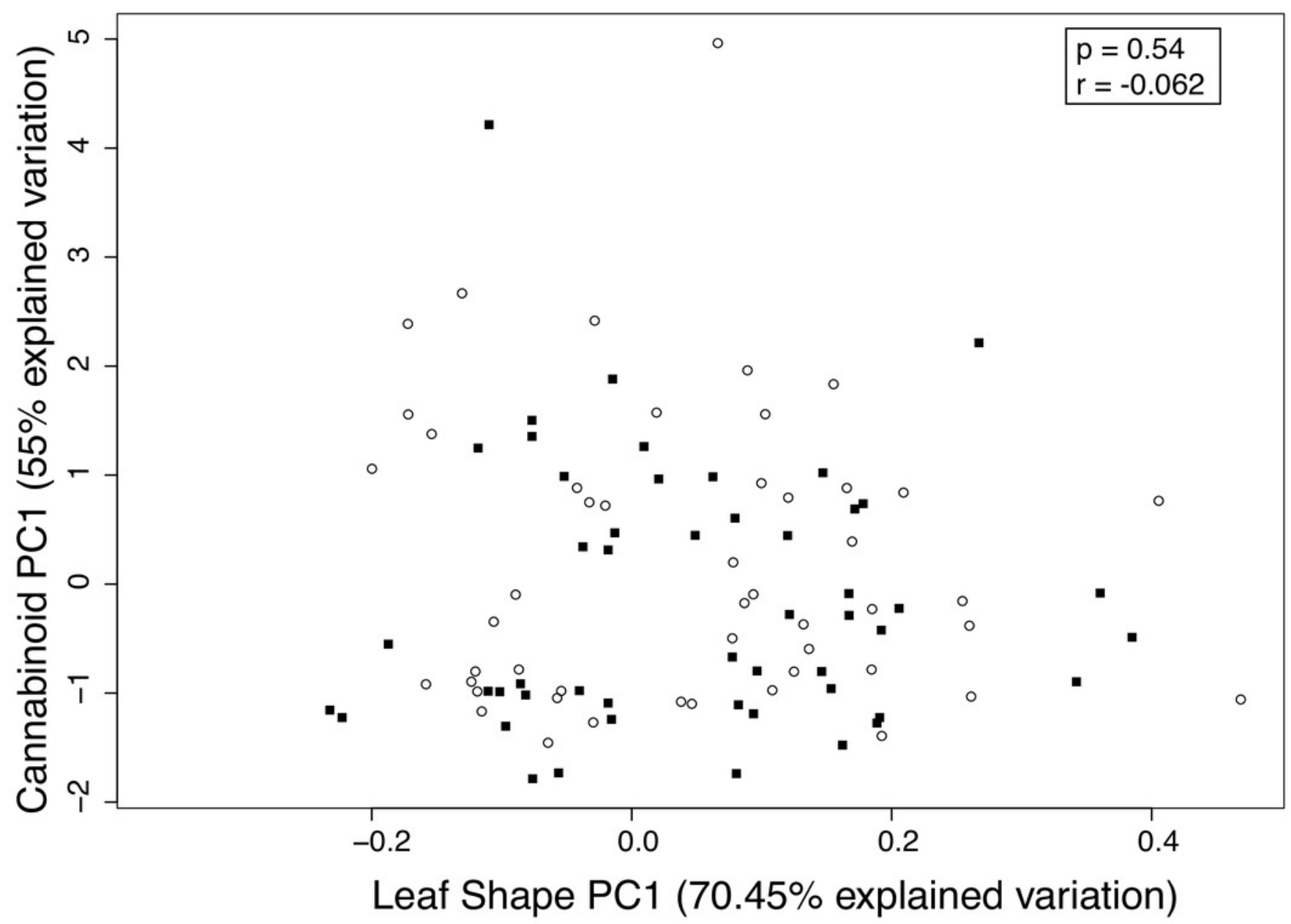

